\title{
Langasite Family Midinfrared Nonlinear Optical Oxide Materials: Structure, Property, and Applications
}

\author{
Haichao Lan, ${ }^{1}$ Fei Liang, ${ }^{2}$ Zheshuai Lin, ${ }^{2}$ Haohai Yu, ${ }^{1}$ Huaijin Zhang, ${ }^{1}$ and Jiyang Wang ${ }^{1}$ \\ ${ }^{1}$ State Key Laboratory of Crystal Materials and Institute of Crystal Materials, Shandong University, Jinan 250100, China \\ ${ }^{2}$ Center for Crystal RßD, Key Lab of Functional Crystals and Laser Technology, Technical Institute of Physics and Chemistry, \\ Chinese Academy of Sciences, Beijing 100190, China
}

Correspondence should be addressed to Haohai Yu; haohaiyu@sdu.edu.cn

Received 24 July 2017; Accepted 3 October 2017; Published 5 November 2017

Academic Editor: Giulio Cerullo

Copyright (C) 2017 Haichao Lan et al. This is an open access article distributed under the Creative Commons Attribution License, which permits unrestricted use, distribution, and reproduction in any medium, provided the original work is properly cited.

\begin{abstract}
Midinfrared (IR) nonlinear optical (NLO) materials with high performance are vital in important technological applications in many civil and military fields. Very recently, langasite family compounds have attracted much attention due to their wide transparency to mid-IR region and ultrahigh laser damage threshold (LDT). In this brief review, three important compounds-LGS, LGN, and LGT - are investigated and analyzed based on available experimental data. The electrooptical (EO) Q-switch and mid-IR OPO applications are summarized in detail. Finally, promising search directions for new metal oxides that have good mid-IR NLO performances are discussed.
\end{abstract}

\section{Introduction}

In 1961, Franken and his colleagues firstly discovered the second-harmonic generation in quartz using a ruby laser beam. And then Bloembergen built a theoretical framework to explain the principle of nonlinear optical parametric generation. Since then, lasers in the human society have been playing an increasingly important role. Nonlinear optical (NLO) materials have a very important significance to convert the laser wavelength into the spectral region and to obtain a high-power laser source. In the past three decades, many useful NLO crystals in near-infrared (IR), visible, and ultraviolet (UV) regions (wavelength from 0.2 to $2 \mu \mathrm{m}$ ) have been developed. In case of the visible region, the $\mathrm{KTP}\left(\mathrm{KTiOPO}_{4}\right)$ crystal has a wide transmission range, high frequency conversion efficiency, good phase-matchable wavelength, high laser damage threshold (LDT), and excellent physical and chemical stability [1]. Regarding UV and deep-UV regions, Chinese scientists have made remarkable contributions. Chen and his collaborators invented the $\mathrm{BBO}$ [2], LBO [3], and KBBF [4] crystals, known as the "Chinese card" crystal. In particular, the KBBF crystal successfully achieves the sixth-harmonic generation of $1.064 \mu \mathrm{m}$, which is the only source of deep-UV laser by direct SHG output [5]. $\mathrm{BiB}_{3} \mathrm{O}_{6}(\mathrm{BIBO})$ crystal possesses large effective nonlinearity, $d_{\text {eff }}$ of $3.7 \mathrm{pm} / \mathrm{V}$, and versatile phase-matching properties. Its large angular and spectral acceptance bandwidths and low spatial walk-off at room temperature thus make BIBO highly attractive for frequency conversion in the visible and UV region [6].

Midinfrared (mid-IR) NLO materials are indispensable for obtaining all-solid-state lasers in the spectral range of 3-20 $\mu \mathrm{m}$ by optical parametric oscillation (OPO) or difference frequency generation (DFG) [7]. Tunable narrow band lasers using NLO crystals have very important applications in many advanced areas of science and technology, such as infrared remote sensing [8], biological tissue imaging [9], environmental monitoring [10], and minimally invasive medical surgery [11]. So far, commercially available midIR NLO crystals are $\mathrm{AgGaS}_{2}$ (AGS), AgGaSe 2 (AGSe), and $\mathrm{ZnGeP}_{2}$ (ZGP). They possess high second-harmonic generation (SHG) coefficients of about $13 \mathrm{pm} / \mathrm{V}, 33 \mathrm{pm} / \mathrm{V}$, and $75 \mathrm{pm} / \mathrm{V}$, respectively [12]. However, these materials have some disadvantages that hinder their use in mid-IR 
laser generation. For example, the laser damage threshold (LDT) values of AGS and AGSe are too small (only about $25 \mathrm{MW} / \mathrm{cm}^{2}$ and $11 \mathrm{MW} / \mathrm{cm}^{2}$ (@1.06 $\mu \mathrm{m}, 35 \mathrm{~ns}$ ), resp.) [13] to bear a high-power pumping source. Meanwhile, the strong two-photon adsorption (TPA) in ZGP resulting from its narrow bandgap $(2.0 \mathrm{eV})$ makes it impossible to use an Nd:YAG laser as the pumping source [14]. Therefore, the current rapid developments of mid-IR lasers urgently demand the discovery of new mid-IR NLO materials with a good performance.

In general, optional midinfrared nonlinear optical materials, in addition to having a noncentral symmetric structure, should meet the following conditions $[15,16]$ : (1) a wider range of transmission bands that can cover important atmospheric transparent windows of 3-5 $\mu \mathrm{m}$; (2) a large nonlinear optical coefficient, because the power of the output light is proportional to the effective nonlinear optical coefficient; (3) high LDT, which essentially depends on the wide bandgap of the material; (4) moderate birefringence $\Delta n$ to achieve a phase-matchable condition; (5) good growth habit and high optical quality; and (6) good physical and chemical stabilities for convenient applications.

In general, metal oxides usually possess a large bandgap and high LDT compared with metal chalcogenides and phosphides. For example, the LDT of LBO and BBO are 45 and $13.5 \mathrm{GW} / \mathrm{cm}^{2}$ (@1064 nm,1ns), which are three orders higher than those of AGS and AGSe. The LDT of KTP is $15 \mathrm{GW} / \mathrm{cm}^{2}$ (@1064 nm, 1ns), consequently larger than that of ZGP $\left(0.03 \mathrm{GW} / \mathrm{cm}^{2}\right)$ [14]. However, commercially available NLO oxides are not transparent in the mid-IR region (3-8 $\mu \mathrm{m})$ limited by high-frequency phonon vibrations. For example, KDP, BBO, and $\mathrm{LBO}$ have an IR cutoff edge of $1.7,3.5$, and $3.2 \mu \mathrm{m}$, respectively [14]. Accordingly, there is a pressing need to develop new metal oxides with relatively low phonon vibrational frequency and wide mid-IR transparency window. Notably, the phonon subsystem plays a negligible role in determining the SHG coefficients of wide bandgap oxides $(>4.0 \mathrm{eV})$ and the "anionic group theory" is still suitable [16]. In addition, the phonon vibrational frequency of $\mathrm{M}-\mathrm{O}$ bond is negatively related to the atomic mass of $\mathrm{M}$ and the bond length of $\mathrm{M}-\mathrm{O}$ bond. Langasite family compounds, with the general formula $\mathrm{A}_{3} \mathrm{BC}_{3} \mathrm{D}_{2} \mathrm{O}_{14}$, have attracted more and more attention in recent years owing to their wide transparency up to $7 \mu \mathrm{m}$ [17], high LDT, and good growth habits. In this brief review, we will introduce the development history of langasite, including crystal structures, physical properties, and growth technology. More importantly, the potential applications of langasites in mid-IR NLO region are summarized.

\section{Structure and Property of Langasite Family}

The langasite family structures belong to the space group P321 (number 150) and they can be described using the general formula $\mathrm{A}_{3} \mathrm{BC}_{3} \mathrm{D}_{2} \mathrm{O}_{14}$ that contains four cationic sites [23]. The $\mathrm{A}$ site is coordinated by eight $\mathrm{O}$ atoms in dodecahedral geometry; the $\mathrm{B}$ site is coordinated by six $\mathrm{O}$ atoms in a distorted octahedral geometry; and the $\mathrm{C}$ and $\mathrm{D}$ sites are coordinated by four $\mathrm{O}$ atoms in distorted tetrahedral geometries (Figure 1), in which the size of the $\mathrm{C}$ site is slightly larger than that of the $\mathrm{D}$ site.

Generally, the langasite family is generally classified into two types depending on the ionic distribution. In ordered langasites, each cationic site is occupied by a different element, while in disordered ones, the same element enters in more than one site. All of them belong to noncentrosymmetric space group and most of them have been grown by Czochralski or Bridgman method successfully. Owing to the length limit of this review, only three important disordered crystals (LGS, LGN, and LGT) are summarized.

Despite being classified into disordered langasite type, LGS, LGN, and LGT have different ionic distributions [24]. In case of LGS, $\mathrm{La}^{3+}$ ions occupy A site, $\mathrm{Ga}^{3+}$ ions occupy the $\mathrm{B}$ and $\mathrm{C}$ sites, plus half of the $\mathrm{D}$ site, and $\mathrm{Si}^{4+}$ ions occupy another half of the D site. In comparison, in case of LGN and LGT, $\mathrm{La}^{3+}$ ions occupy the A site, $\mathrm{Nb}^{5+} / \mathrm{Ta}^{5+}$ ions occupy half of the $\mathrm{B}$ site, and $\mathrm{Ga}^{3+}$ ions occupy $\mathrm{C}$ and $\mathrm{D}$ sites, plus half of the B site. Different ovalent and aliovalent substitutions in a given structure are quite interesting in themselves and could, perhaps, also result in improved structural and piezoelectric properties. According to the structure-property relationship, different physical properties are expected in these three crystals, such as piezoelectric, electrooptical, and secondorder NLO coefficients.

Langasite (LGS) and several isomorphic compounds have attracted the attention of researchers due to their special spectroscopic and piezoelectric properties since the early 1980s [25]. Since they show no phase transitions up to their melting points of approximately $1470^{\circ} \mathrm{C}$, the material is considered to be able to replenish or even replace $\alpha$ quartz as surface acoustic wave (SAW) filters and sensors for pressure, force, and acceleration at high temperatures [26]. In 1983, Kaminskii et al. reported the laser properties of $\left(\mathrm{La}_{1-x} \mathrm{Nd}_{x}\right)_{3} \mathrm{Ga}_{5} \mathrm{SiO}_{14}$ (Nd:LGS) crystal for the first time and made a preliminary estimate about the crystal of some other uses [27, 28]. Subsequently, the growth and laser properties of LGS, Nd:LGS, and Nd-doped $\mathrm{Ca}_{3} \mathrm{Ga}_{2} \mathrm{Ge}_{4} \mathrm{O}_{14}$ (CGG) and $\mathrm{Sr}_{3} \mathrm{Ga}_{2} \mathrm{Ge}_{4} \mathrm{O}_{14}$ (SGG) were studied in 1983, and their properties such as optics, piezoelectricity, elasticity, and typical values were also measured [29].

Langasite family compounds have only two independent thermal expansion coefficients, $\alpha_{11}$ and $\alpha_{33}$, whose directions are perpendicular and parallel to $z$-axis. The thermal expansion coefficients are 5.8 and $3.9 \cdot 10^{-6} / \mathrm{K}$, respectively. In case of LGS, it is clear that the thermal expansion of the crystal is anisotropic along the different crystallographic axes, but the anisotropy is not very strong and smaller than that of KDP $[30,31]$. In addition, the thermal expansion coefficients $\alpha_{11}$ and $\alpha_{33}$ of LGN (LGT) are $6.1(6.3)$ and $4.8(4.6) \cdot 10^{-6} / \mathrm{K}$, respectively [30]. Moreover, the thermal conductivities of LGS, LGN, and LGT are $1.3,1.4$, and $1.2 \mathrm{~W} / \mathrm{m} \cdot \mathrm{K}$ along the $X$ direction, respectively, while they are $1.9,1.7$, and $1.7 \mathrm{~W} / \mathrm{m} \cdot \mathrm{K}$ along the $Z$-direction [32]. These values are slightly lower than those of $\mathrm{LiNbO}_{3}(4.4 \mathrm{~W} / \mathrm{m} \cdot \mathrm{K})$ and $\mathrm{KTP}(2 \mathrm{~W} / \mathrm{m} \cdot \mathrm{K})$. Furthermore, the specific heat of three crystals is $0.45,0.50$, and $0.36 \mathrm{~J} /(\mathrm{g} \cdot \mathrm{K})$, which is comparable with $\mathrm{KTP}(0.68 \mathrm{~J} /(\mathrm{g} \cdot \mathrm{K}))$ 


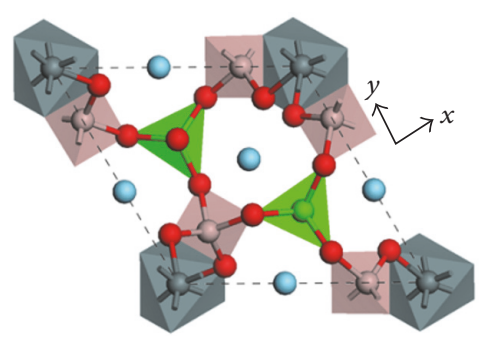

(a)
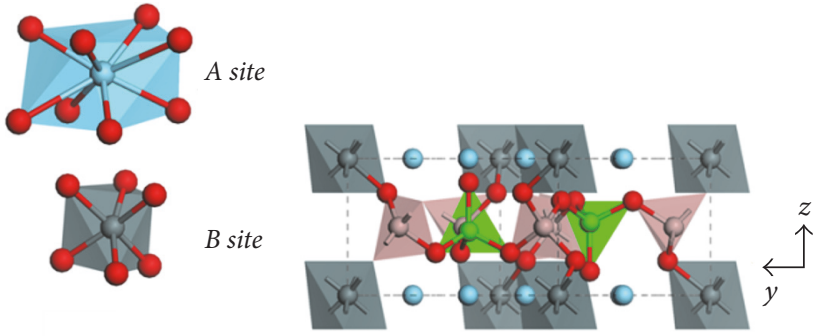

(b)

Figure 1: Crystal structure of $\mathrm{A}_{3} \mathrm{BC}_{3} \mathrm{D}_{2} \mathrm{O}_{14}$ : (a) three-dimensional framework viewed along the $c$-axis; (b) three-dimensional framework viewed along the $a$-axis; the local distorted polyhedron of different cation sites, including decahedral A site, octahedral B site, tetrahedral C site, and tetrahedral D site.

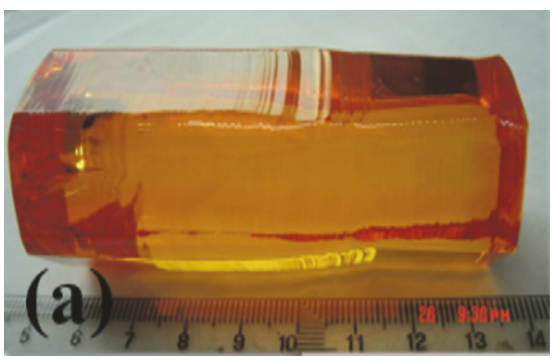

(a)

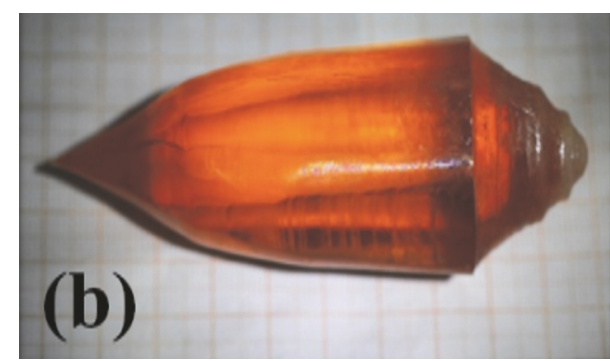

(b)

FIGURE 2: The photos of LGS and LGN crystals grown by Czochralski's method.

and $\mathrm{LiNbO}(0.65 \mathrm{~J} /(\mathrm{g} \cdot \mathrm{K}))$. From the above results, it is shown that the thermal properties of langasite compounds are suitable for optical applications.

The LGS series crystals can be grown by Czochralski's method and the length of the largest crystal is more than $10 \mathrm{~cm}$. The crystal photos are displayed in Figure 2. It is clear that all of them are transparent and are of optical high quality. Recently, our group measured their transmission spectra, as displayed in Figure 3. Clearly, all the three crystals have high transparency ranging from 0.5 to $5 \mu \mathrm{m}[17,22]$, which covers the important atmosphere transparency window. In addition, the transparent region of LGS is slightly narrowed compared with LGN and LGT, which can be attributed to high-frequency Si-O bond vibrations. There are strong and narrowly polarized absorption peaks at $1.85 \mu \mathrm{m}$ and a small absorption peak at $3 \mu \mathrm{m}$, due to oxygen defects and Ga$\mathrm{O}$ bond vibrations, respectively. The wide transparency of langasite compounds indicates that they are potential mid-IR optical materials. However, although their SHG coefficients were measured many years ago [33], few people focus on the NLO applications of LGS. In 2014, Boursier's group reported for the first time a complete study of mid-IR NLO properties of LGT [22], and then Yu's research group followed up and investigated the LGN crystal, in which signal beam 1.43 and idler beam $4.14 \mu \mathrm{m}$ were obtained [22]. Now, we will introduce the mid-IR applications of langasite family in detail from two aspects: electrooptic (EO) Q-switched devices and NLO optical parametric process.

\section{Electrooptical (EO) Q-Switch Applications}

Electrooptical (EO) effect is the phenomenon where the refractive index of the crystal changes with the electric field. The change in refractive index proportional to the external electric field is called the Pockels effect, while the change in the ratio of the square of the external electric field is called the Kerr effect. The EO effect can be regarded as a kind of nonlinear optical effect, and all non-centrally symmetric crystals (except 432 point group) have a linear EO effect. Although the refractive index of the EO effect crystal is generally not changed under the action of the electric field, it is sufficient to cause the propagation of light to change in the crystal, so that we can achieve the purpose of photoelectric signal conversion or mutual modulation by changing the external field. EO effect has a wide range of applications in laser technology, EO crystal commonly used as Q-switch, EO modulator, EO shutter, and so on [31]. In particular, EO Qswitch is an important optical device to achieve high-energy laser output impulse lasers.

A good EO crystal should satisfy the following conditions: large EO coefficient, wide band transmission range, high LDT, and good growth habit to obtain a large-size crystal in favor of EO Q-switched applications. So far, commercially available EO crystals are mainly $\mathrm{LiNbO}_{3}$, KTP, RTP, BBO, and DKDP crystals [34]. The large-size DKDP and BBO crystals are difficult to grow, whereas LN has a relatively low optical damage threshold (only $100 \mathrm{MW} / \mathrm{cm}^{2}$ ). Moreover, 


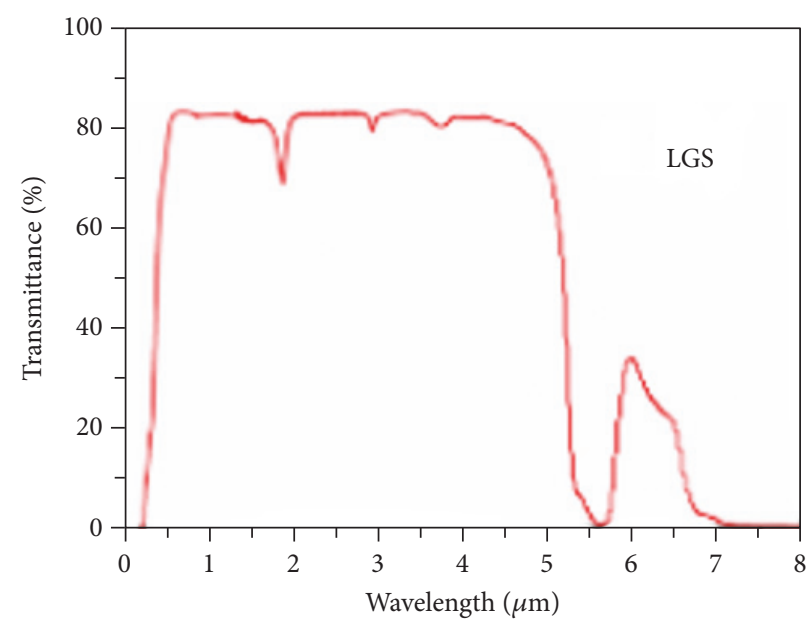

(a)

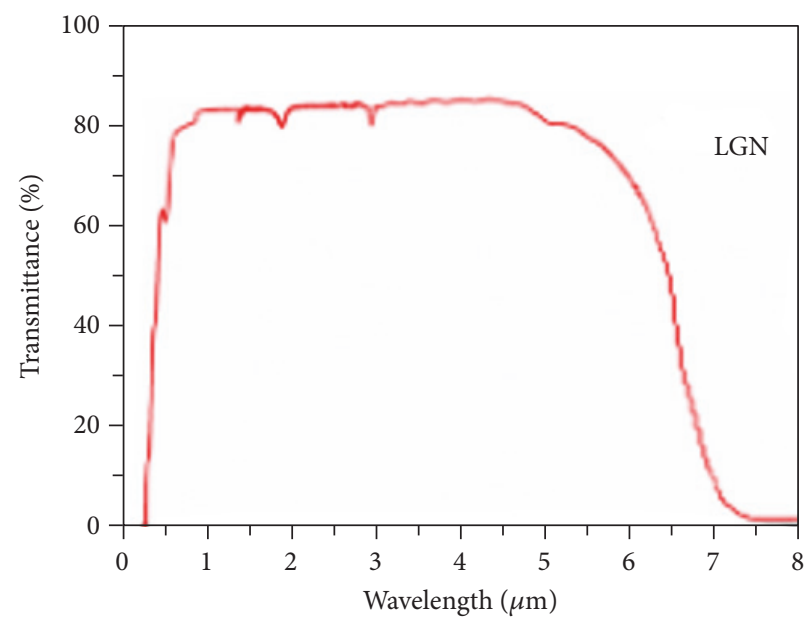

(b)

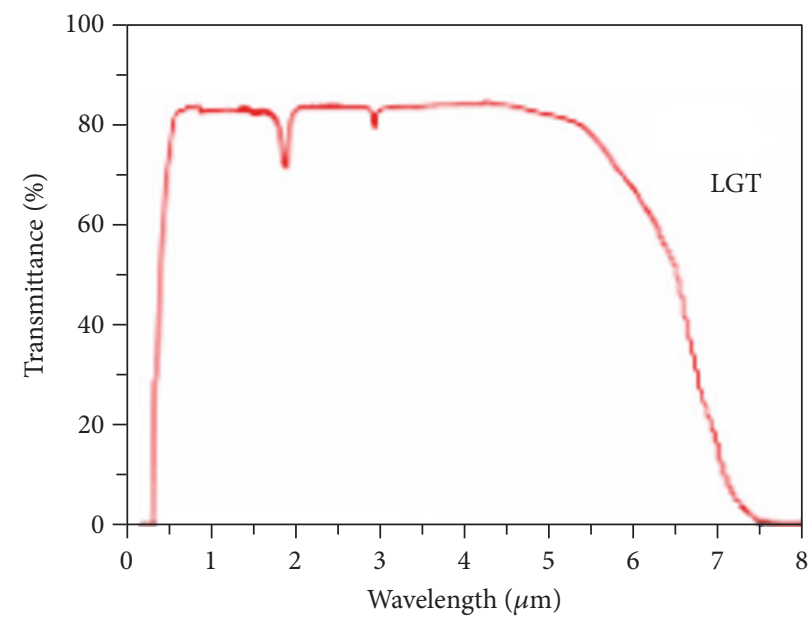

(c)

FIGURE 3: The transmission spectra of (a) LGS, (b) LGN, and (c) LGT crystal.

KTP is a biaxial crystal and the static birefringence must be compensated when used as Q-switch devices. Moreover, the $z$-axis electrical resistance of KTP is too low to bear highpower lasers so as to cause gray-tracking damage. Recently, high-resistance RTP crystals have been used for EO Q-switch applications [35]. At present, no better crystals have been found to fulfil the demand of high-performance EO Qswitches, especially those lasers that generate high repetition rates.

Since 2002, LGS has been promoted as a potential EO crystal owing to its large EO coefficients, high LDT, and excellent physicochemical stability [31]. The linear EO properties of LGS crystal were measured to be $\gamma_{11}=-2.68 \mathrm{pm} / \mathrm{V}$ and $\gamma_{41}=1.22 \mathrm{pm} / \mathrm{V}$ in 2002 [24]. And then, those values of LGS were refined to be 2.3 and $1.8 \mathrm{pm} / \mathrm{V}$ by our group [36]. Despite being lower than the DKDP and $\mathrm{LiNbO}_{3}$ crystal, the halfwave voltage can be adjusted by changing the aspect ratio of light propagating length and crystal thickness of the applied voltage. Accordingly, the research of EO properties of LGS has become a new scientific hotspot. LGS exhibits optical activity, which means that the light polarization direction is rotated as it propagates through the crystal. It has been determined that the effect of optical activity on the electrooptic process can be eliminated by reversing the effect, which means that light propagating along the optic $z$-axis should be reversed to propagate along the $z$-axis. In addition, the EO coefficients of LGN and LGT were also measured by Stade's group [24]. They have a comparable $\gamma_{11}(-2.62$ and $-2.82 \mathrm{pm} / \mathrm{V})$ with LGS $(-2.68 \mathrm{pm} / \mathrm{V})$. Notably, their optical activities are stronger than that of LGS, which is slightly unfavorable for practical applications. Subsequently, many researchers mainly focused on potential applications of LGS crystals. Now, we review three typical applications of IR coherent generation using LGS EO Q-switched devices.

3.1. 1.064 $\mu \mathrm{m}$ Laser Generation. In 2003, LGS Q-switched devices have been successfully used in $1.064 \mu \mathrm{m} \mathrm{Nd:YAG} \mathrm{laser}$ generation, where $350 \mathrm{~mJ}$ pulse energy with $7.8 \mathrm{~ns}$ pulse width output was obtained [36]. In 2009, Q-switched Nd:YVO lasers properties of $30 \mathrm{kHz}$ with LGS electrooptic modulator were studied in detail [37]. End-pumped by laser diodes, an 


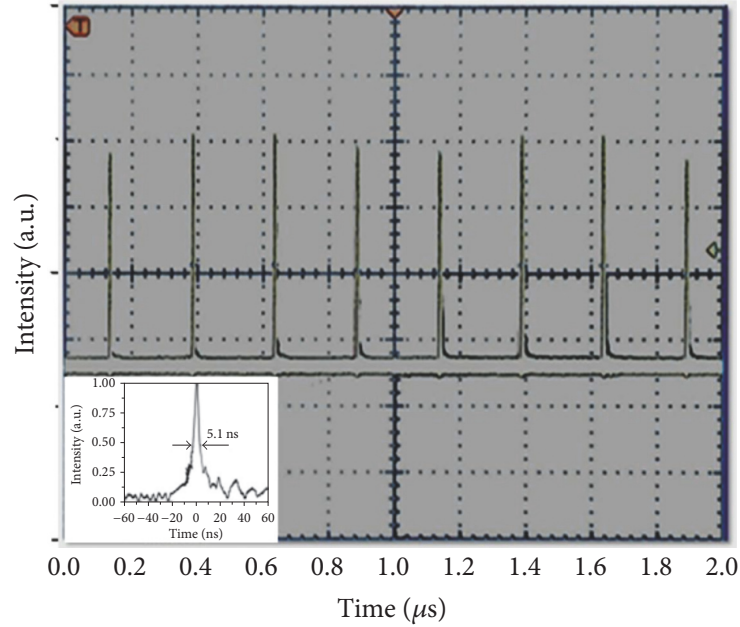

FIgURE 4: Pulse train with a repetition rate of $200 \mathrm{kHz}$ in the LGS electrooptic Q-switched Nd:LuVO 4 laser. Inset: pulse profile with the shortest pulse width of $5.1 \mathrm{~ns}$. Reprinted with permission from [18]. Copyright 2016 Springer Nature Group.

average output power of $6.2 \mathrm{~W}$ with the pulse width of $9.1 \mathrm{~ns}$ was obtained.

In order to obtain a shorter pulse width of $1.064 \mu \mathrm{m}$ laser, Ma designed Q-switched lasers based on the theory of "odd transport time" and Nd:LuVO 4 crystal in 2016 [18]. The LGS crystal with a size of $5 \times 5 \times 25 \mathrm{~mm}^{3}$ was cut along the $z$-axis. By optimizing parameters, a stable LGS Q-switched laser was designed with an average output power of $4.39 \mathrm{~W}$, corresponding to a slope efficiency of $29.4 \%$, and with a minimum pulse width of $5.1 \mathrm{~ns}$ (Figure 4 ). The performance of the pulse laser with $200 \mathrm{kHz}$ repetition rate is comparable to the $\mathrm{BBO} \mathrm{Q}$-switched laser with $200 \mathrm{kHz}$ repetition rate and better than the DKDP $(10 \mathrm{kHz})$ [38], LN $(7 \mathrm{kHz})$ [39], and other LGS (30 kHz) [37] Q-switched lasers. Additionally, the observed pulse width of $5.1 \mathrm{~ns}$ is also smaller than the narrowest pulse width obtained with DKDP (20 ns) [40], LN (12 ns) [39], and other LGS (7.8 ns) [37] Q-switch lasers. This work represents the highest repetition rate observed so far in the LGS Q-switched laser regime with a pulsed laser wavelength ranging from 1.0 to $3.0 \mu \mathrm{m}$. These results indicate that LGS can be used as a high repetition rate Q-switch and is free of piezoelectric ringing effects at least at a repetition rate of $200 \mathrm{kHz}$ and that it can provide a practical Q-switched laser with a tunable high repetition rate for many applications.

3.2. 2.09 $\mu \mathrm{m}$ Laser Generation. A flash lamp pumped $2.09 \mu \mathrm{m}$ $\mathrm{Cr}$,Tm,Ho:YAG laser utilizing a self-grown $\mathrm{La}_{3} \mathrm{Ga}_{5} \mathrm{SiO}_{14}$ crystal as the EO Q-switch generator is proposed and demonstrated for the first time [19]. A Cr,Tm,Ho:YAG rod with a diameter of $5 \mathrm{~mm}$ and a length of $127 \mathrm{~mm}$ was used as a gain medium. The doping concentrations of the $\mathrm{Cr}, \mathrm{Tm}, \mathrm{Ho}$ :YAG laser crystal are $1.5 \%, 5.8 \%$, and $0.35 \%$ for $\mathrm{Cr}, \mathrm{Tm}$, and Ho, respectively. To obtain high output energy, a quartz quarter-wave plate was utilized to compensate for the losses caused by thermal depolarization. A $z$-cut LGS crystal was

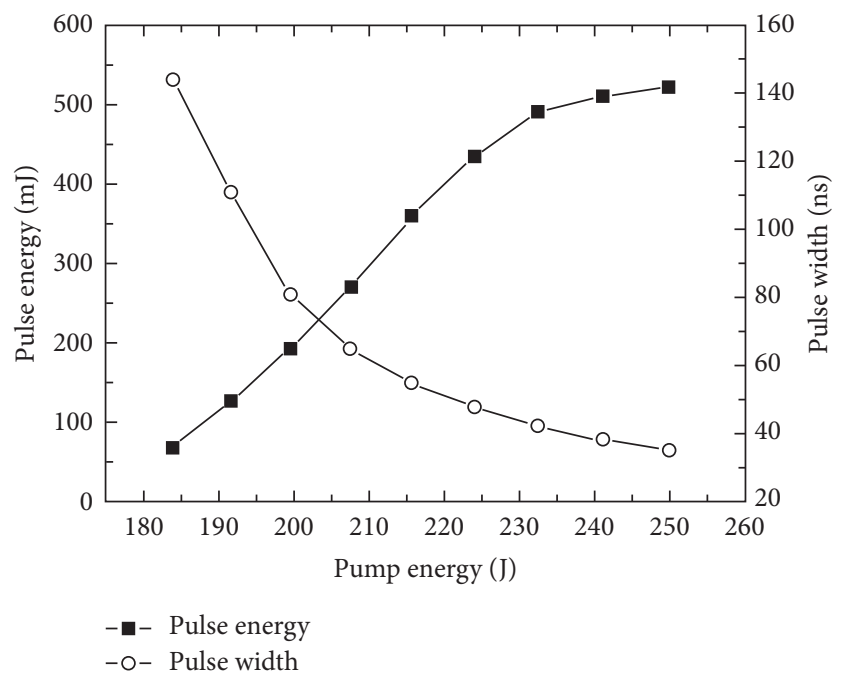

FIGURE 5: Output energy and pulse width as a function of the pump energy in LGS EO Q-switched Cr,Tm,Ho:YAG laser. Reprinted with permission from [19]. Copyright 2012 Optical Society of America.

designed and cut in a size of $7 \times 7 \times 45 \mathrm{~mm}^{3}$ to decrease the quarter-wave voltage. The Q-switch operates in transversefield configuration in which an electric field was applied along the $x$-axis while the light beam propagated along the $z$-axis of the LGS crystal. The LGS EO Q-switch can operate properly when a voltage of $4100 \mathrm{~V}$ is applied.

After the opening delay time of Q-switch was optimized and the thermal depolarization was compensated, $520 \mathrm{~mJ}$ pulse energy with $35 \mathrm{~ns}$ pulse width was obtained at a pump energy of $250 \mathrm{~J}$ at $3 \mathrm{~Hz}$ and corresponding peak power of 14.86 MW. To the best of our knowledge, this is the largest Q-switched pulse energy in $2 \mu \mathrm{m}$ wavelength range without amplification. As shown in Figure 5, the pulse width decreases with the increase of the pump energy. The output energy keeps increasing and tends to be saturated. The output energy and pulse width instability are $4.9 \%$ and $5.3 \%$, respectively. In the experiments, by optimizing the Q-switch opening delay time to $750 \mu \mathrm{s}$, the highest peak power has been achieved, which corresponds to a Q-switched-to-normal-mode energy extraction efficiency as high as $66.3 \%$. Compared with RTP Q-switch, relatively low pulse energy of $42 \mathrm{~mJ}$ was obtained, which was limited by the damage threshold. Accordingly, the LGS crystal is an excellent candidate as a Q-switch generator to obtain higher energy.

3.3. $2.79 \mu \mathrm{m}$ Laser Generation. The $2.79 \mu \mathrm{m}$ Cr,Er:YSGG laser exhibits potential for medical applications due to its good water absorption. On the other hand, a $2.79 \mu \mathrm{m}$ pulsed laser with high peak power and high pulse energy can be used as a pump source for optical parametric oscillators (OPO) to obtain 3-12 $\mu \mathrm{m}$ midinfrared laser radiation. Since RTP has absorption around $2.8 \mu \mathrm{m}$, it cannot be used as a Qswitching crystal for this wavelength [41]. To date, LN has been successfully demonstrated as a Q-switching crystal at $2.79 \mu \mathrm{m}$ in a relatively high-energy laser; however, its LDT is too low $\left(100 \mathrm{MW} / \mathrm{cm}^{2}\right)$. Therefore, we consider that LGS is an 


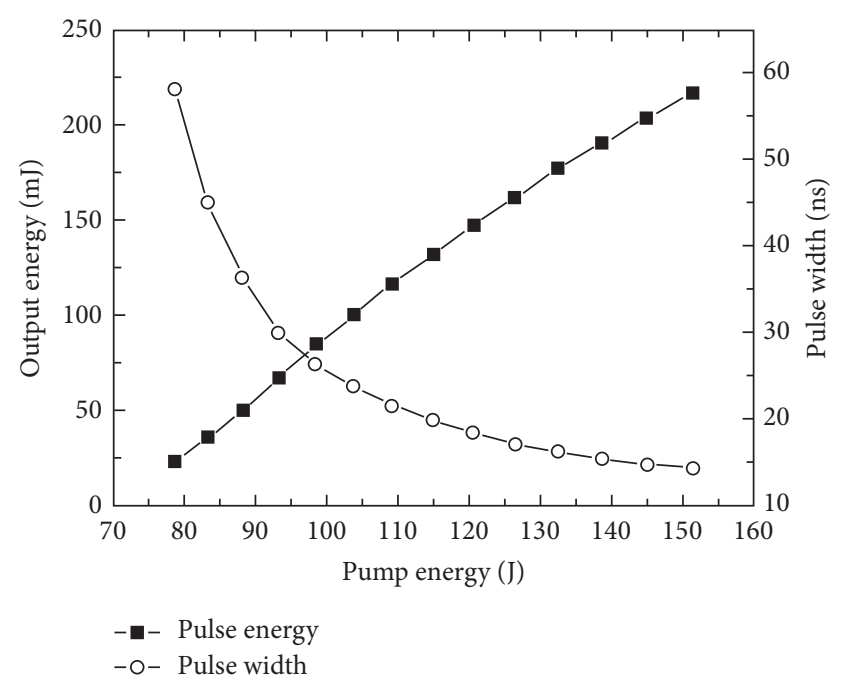

FIgURE 6: Output energy and pulse width as a function of pump energy in LGS EO Q-switched Cr,Er:YSGG laser. Reprinted with permission from [20]. Copyright 2013 Optical Society of America.

ideal candidate for an EO Q-switch operating at the $2.79 \mu \mathrm{m}$ wavelength [20].

To verify the Q-switching performance of LGS at the $2.79 \mu \mathrm{m}$ wavelength, a Cr,Er:YSGG crystal was employed as a gain medium to form a Q-switched laser system. The Cr,Er:YSGG laser rod has a diameter of $4 \mathrm{~mm}$ and a length of $86 \mathrm{~mm}$ and is doped with $2 \% \mathrm{Cr}^{3+}$ and $30 \% \mathrm{Er}^{3+}$. A $z$-cut LGS crystal was designed and cut in a size of $7 \times 7 \times 50 \mathrm{~mm}^{3}$ to decrease the quarter-wave voltage.

After optimizing the laser system mentioned above, a Qswitched giant pulse with $216 \mathrm{~mJ}$ pulse energy and $14.36 \mathrm{~ns}$ pulse width was obtained for pump energy of $151 \mathrm{~J}$ at $3 \mathrm{~Hz}$. The Q-switched-to-normal-mode energy extraction efficiency is $63.5 \%$, and its corresponding peak power is $15 \mathrm{MW}$. To the best of our knowledge, the obtained pulse energy and pulse width are the best results among pulsed lasers working at around $2.79 \mu \mathrm{m}$ reported to date. These should be attributed to the high damage threshold of the EO Q-switch crystal employed in our experiments and the high pump energy, so high pulse energy with short pulse width can be obtained in such a high gain laser system. As shown in Figure 6, the pulse width decreases with the increase of the pump energy, and the output energy keeps increasing without saturation in our experiments, implying that it is possible to further increase the output energy by increasing the pump energy. The damage threshold of LGS at $2.79 \mu \mathrm{m}$ was measured to be about $750 \mathrm{MW} / \mathrm{cm}^{2}$ by focusing this Q-switched laser on the LGS sample.

In summary, LGS has good prospects for EO Q-switching crystals operating at near-IR and mid-IR region and LGSbased Q-switched lasers will drive the development of short pulses and high-energy laser devices.

\section{Mid-IR NLO Applications}

The research on the LGS system in the piezoelectric application has been very mature, but this is a new application area where NLO applications are rare and further research and development are necessary. Under the Kleinmann assumption, there is only one independent coefficient $d_{11}$. The nonlinear coefficients and coherence lengths of langasite families were measured for the first time in 1989, taking $\alpha$ $\mathrm{SiO}_{2}$ as a reference [33]. And then, the NLO investigations of these compounds have been neglected for a long time. Recently, the langasite families have been reported to have better midinfrared nonlinear properties compared to KTP, especially in mid-IR atmosphere windows $(3-5 \mu \mathrm{m})$ [17, 22]. Langasite's broad transparent region, moderate effective nonlinear coefficient, high damage threshold, small spatial walk-off, large size scalability, and good thermomechanical properties make it very promising as a mid-IR nonlinear crystal.

4.1. $\mathrm{La}_{3} \mathrm{Ga}_{5} \mathrm{SiO}_{14}$ (LGS). The NLO investigations for LGS have already begun since 1989 [33]. In 1997, the SHG $d_{11}$ of LGS was also examined to be two times larger than that of quartz by the Maker Fringe method firstly [42]. In addition, the parameters in the Sellmeier equation for LGS are accurately obtained on the basis of the measurement of the refractive indices in the region of $500 \mathrm{~nm}$ to $2500 \mathrm{~nm}$, and the phase-matching of LGS was investigated [42]. Owing to the small birefringence $(<0.015)$, it was revealed that LGS cannot be used to obtain the phase-matching Type I or Type II for SHG in the range of $500 \mathrm{~nm}-2500 \mathrm{~nm}$. Recently, our group grew large-size LGS by Czochralski's method. It has a high transparency range from 0.5 to $5 \mu \mathrm{m}$, plus an extra window ranging from 6 to $7 \mu \mathrm{m}$. The nonlinear coefficient $d_{11}$ of LGS was refined to be $1.86 \mathrm{pm} / \mathrm{V}$, which is consistent with Kaminskii et al.s result ( $1.7 \mathrm{pm} / \mathrm{V})$. Compared with LGN and LGT, SHG coefficient of LGS is relatively small.

4.2. $\mathrm{La}_{3} \mathrm{Ga}_{5.5} \mathrm{Nb}_{0.5} \mathrm{O}_{14}(\mathrm{LGN})$. The linear and nonlinear optical properties of LGN were studied by our group in detail [17]. The transmission spectra of LGN are depicted in Figure 2(b). LGN is transparent between 0.28 and $7.4 \mu \mathrm{m}$.

As can be seen from Figure 7(a), $n_{e}$ is always larger than $n_{o}$, which means LGN is a positive uniaxial crystal. A large birefringence $\Delta n \sim 0.03$ is favorable for phase-matching condition. The nonlinear coefficient $d_{11}$ of LGN was $3.0 \pm$ $0.1 \mathrm{pm} / \mathrm{V}$ at $532 \mathrm{~nm}$, measured by Maker Fringe method (Figure 7(b)). Moreover, the optical damage threshold of LGN is $1.41 \mathrm{GW} / \mathrm{cm}^{2}$ [17], which is much larger than that of KTP and $\mathrm{LiNbO}_{3}$.

A Type II OPG pumped at $1.064 \mu \mathrm{m}$ was implemented, using an LGN slab with dimensions of $4 \times 4 \times 21 \mathrm{~mm}^{3}$. It was cut in the $(y, z)$ plane along the phase-matching direction $\left(\theta_{\mathrm{PM}}=52^{\circ}, \varphi_{\mathrm{PM}}=90^{\circ}\right)$, polished for optical quality, and uncoated. Figure 8 shows the recorded signal and idler spectrum of the Type II LGN-OPG ranging from $1.06 \mu \mathrm{m}$ to $4.7 \mu \mathrm{m}$. The emission of a signal beam at $\lambda_{s}=1.43 \mu \mathrm{m}$ and an idler beam at $\lambda_{i}=4.14 \mu \mathrm{m}$ is expected in this direction by using our phase-matching curves [17]. From these results, it can be seen that LGN is a promising highenergy crystal which can grow into a large size. It can also be applied to propagation in the range of atmospheric 


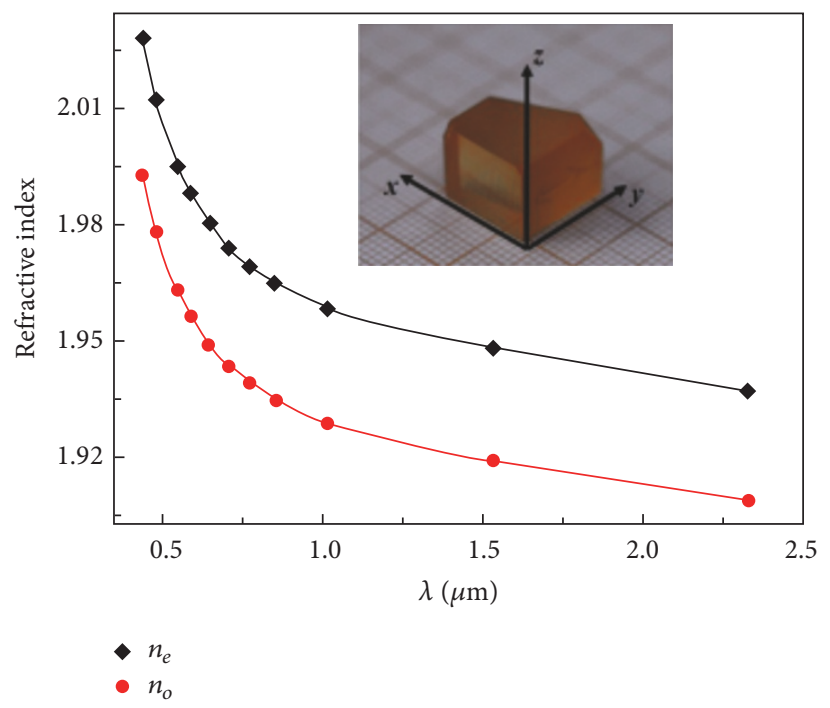

(a)

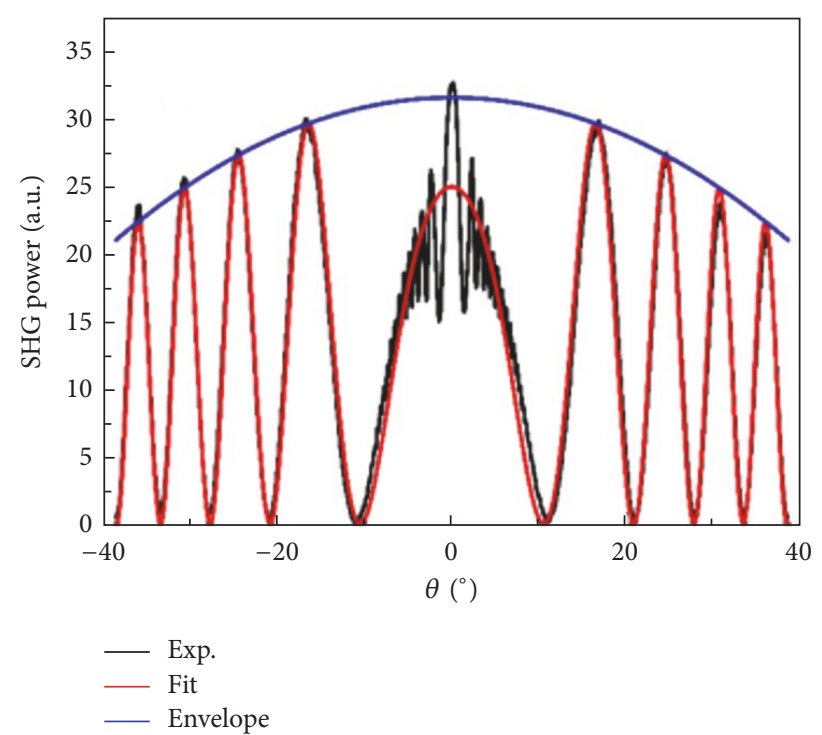

(b)

Figure 7: (a) Measured principal refractive indices $n_{o}$ and $n_{e}$ plotted as a function of wavelength. The insert picture shows the oriented centimeter-size LGN prism. (b) The Maker Fringes pattern $d_{11}$ of LGN. Reprinted with permission from [17]. Copyright 2016 Optical Society of America.

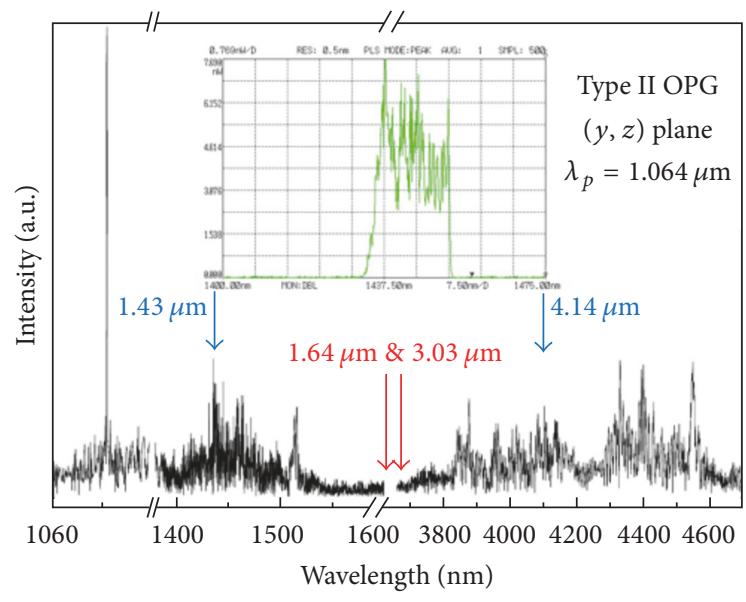

FIGURE 8: Recorded spectra at the output of LGN-OPG pumped by Nd:YAG laser at $1.064 \mu \mathrm{m}$. Reprinted with permission from [17]. Copyright 2016 Optical Society of America.

bandwidth $(3-5 \mu \mathrm{m})$, such as radars. Further experiments will be devoted to optical parametric oscillation (OPO), using the proper cavity design and coating of the LGN crystal faces.

Very recently, LGN exhibits a favorable set of optical properties for the application in mid-IR optical parametric amplification (OPA) [43] or optical parametric chirped pulse amplification (OPCPA) [44]. Ma's group theoretically evaluated the performance of LGN as the nonlinear crystal of a midinfrared OPA and calculated the key parameters of wide bandwidth OPCPA [21]. LGN can support broadband amplification across its entire midinfrared transparent region up to $6 \mu \mathrm{m}$, outperforming other commonly used midinfrared crystals in terms of gain bandwidth.
TABLE 1: Calculation parameters for different crystals in the $2.108 \mu \mathrm{m}$ OPA pumped at $1.064 \mu \mathrm{m}$.

\begin{tabular}{lcccc}
\hline Crystal & $\theta$ (deg.) & $d_{\text {eff }}(\mathrm{pm} / \mathrm{V})$ & $D 2\left(\mathrm{fs}^{2} / \mathrm{mm}\right)$ & Walk-off $($ deg. $)$ \\
\hline LGN & 49.04 & 1.73 & -31.90 & 0.80 \\
$\beta$-BBO & 21.92 & 2.41 & -222.84 & 3.02 \\
LiNbO $_{3}$ & 44.61 & 3.97 & -154.06 & 2.06
\end{tabular}

The Type I degenerate OPA at $2.108 \mu \mathrm{m}$ is firstly calculated, with the results summarized in Figure 9 and Table 1. Comparisons are made between the crystals of LGN, $\beta$-BBO, and $\mathrm{LiNbO}_{3}$. As shown by the dashed lines in Figure 9(a), LGN has the smallest phase mismatch across the spectral range from $1.5 \mu \mathrm{m}$ to $3 \mu \mathrm{m}$. On average, the phase mismatches in $\beta$-BBO and $\mathrm{LiNbO}_{3}$ are larger than that in LGN by $\sim 6.5$ and $\sim 4.5$ times, respectively. This implies that LGN can support a much larger gain bandwidth (Figure 9(a)). Compared under the same gain of 1000, the gain bandwidth of LGN is still much larger than those of $\mathrm{LiNbO}_{3}$ and $\beta$ BBO (Figure 9(b)). Further calculations demonstrate that the zero-dispersion wavelengths of $\mathrm{LGN}, \mathrm{LiNbO}_{3}$, and $\beta$-BBO are located at $1.99 \mu \mathrm{m}, 1.89 \mu \mathrm{m}$, and $1.43 \mu \mathrm{m}$, respectively. We can see that the zero-dispersion wavelength of LGN is closest to the desired signal wavelength of $2.109 \mu \mathrm{m}$, so the LGN OPA has the smallest $D 2$, as shown in Table 1 . This is why LGN can support a so much large bandwidth.

In addition, the $3.5 \mu \mathrm{m}$ noncollinear OPA pumped at $1.054 \mu \mathrm{m}$ of LGN was also calculated with $\mathrm{LiNbO}_{3}$ and KTA as comparison. In this spectral band, the LGN still exhibits the smallest phase mismatch and thus the largest gain bandwidth. From the above calculation and analysis, it can be seen that LGN is suitable for broadband in the infrared OPA excellent 


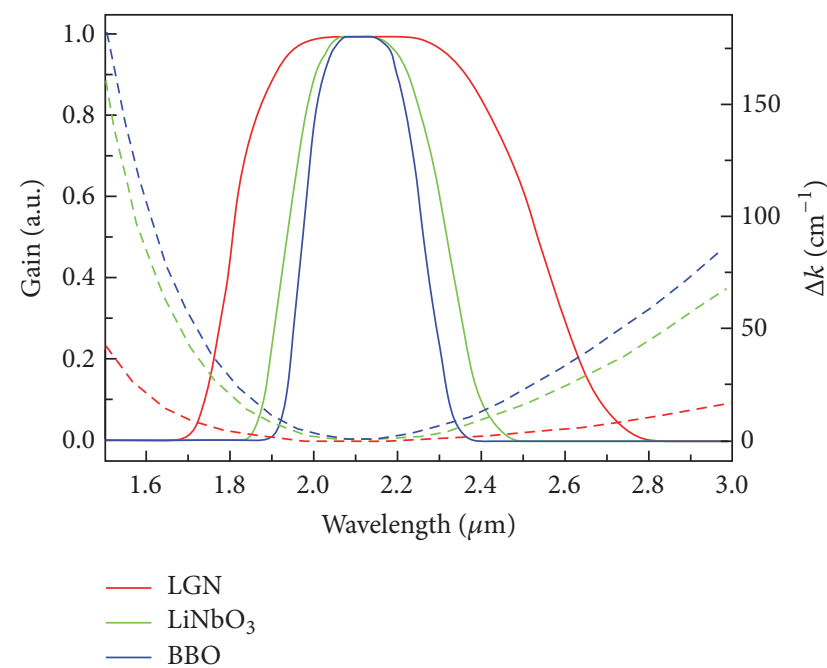

(a)

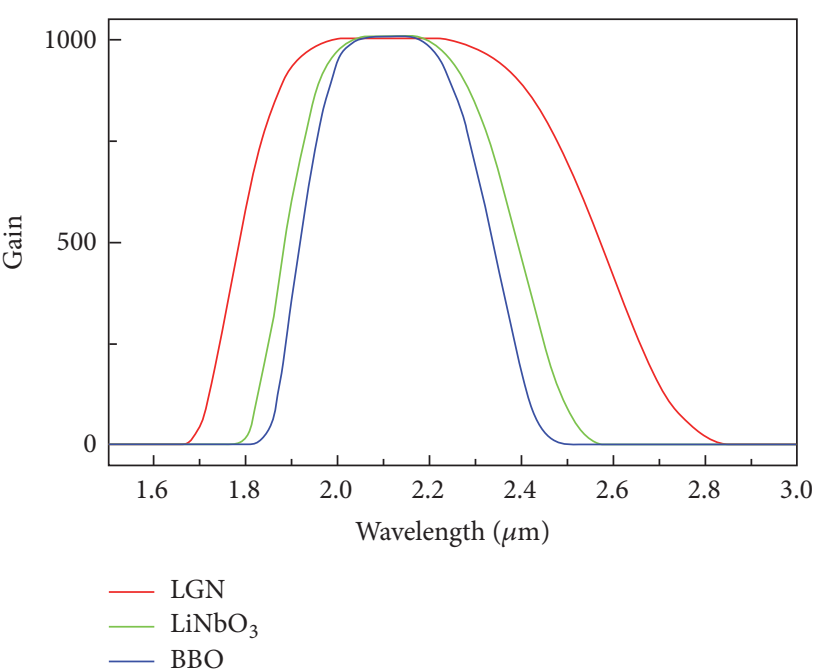

(b)

Figure 9: (a) Gain spectra (solid curves) and phase mismatches (dashed curves) for a $2.108 \mu \mathrm{m}$ degenerate OPA pumped at $1.054 \mu \mathrm{m}$. Red, green, and blue curves correspond to LGN and LiNbO3 crystals. (b) Comparisons under the same gain for different crystals. Reprinted with permission from [21]. Copyright 2016 Optical Society of America.

crystal. Its tolerance to phase mismatch is superior to other commonly used crystals. And its zero-dispersion wavelength $(\sim 2 \mu \mathrm{m})$ in the midinfrared region creates more hope for a wider gain bandwidth.

Compared with OPA, near-infrared laser pumped OPCPA is more promising for producing high-energy midinfrared lasers without causing significant nonlinear pulse distortion or causing damage. Here, the broadband mid-IR OPCPAs based on LGN were also investigated by theoretical calculations. Two types of mid-IR OPCPAs pumped at $1.054 \mu \mathrm{m}$ are considered in this section: one is the Type I degenerate OPA at $2.108 \mu \mathrm{m}$ and the other is the Type II collinear OPA at $4.44 \mu \mathrm{m}$. Figure 9 summarizes the simulation results for the Type I degenerate OPCPA at $2.108 \mu \mathrm{m}$. Due to a smaller $d_{\text {eff }}$, the needed LGN length for peak conversion is nearly two times the $\mathrm{LiNbO}_{3}$ length (Figure 10). For chirped seeding pulse, different spectral components are distributed at different temporal positions. Therefore, the larger gain bandwidth brings about a more efficient signal amplification and a stronger pump depletion, as shown in Figures $10(\mathrm{a})$ and $10(\mathrm{~b})$. The positions marked by points $\mathrm{A}$ and $\mathrm{B}$ are used to carry out the following calculations, which correspond to $15.55 \mathrm{~mm}$ long LGN and an $8.11 \mathrm{~mm}$ long LiNbO3. As expected, the LGN-based OPCPA exhibits an ultrabroad gain bandwidth as large as about $900 \mathrm{~nm}$ (FWHM), as shown in Figure 10(c). By contrast, the gain bandwidth of the $\mathrm{LiNbO}_{3}$-based OPCPA is limited to about $550 \mathrm{~nm}$. For the LGN-based OPCPA, a dispersion compensation of $108 \mathrm{fs}^{2}$ leads to the shortest pulses with a duration of $13.6 \mathrm{fs}$, very close to its Fourier transform limit (Figure 10(d)). It is sub-two cycles at $2.108 \mu \mathrm{m}$. For the $\mathrm{LiNbO}_{3}$-based OPCPA, the OPP can also be compensated by introducing a larger dispersion of $243 \mathrm{fs}$ to achieve the
near-Fourier-transform-limited pulse duration of $21 \mathrm{fs}$ (three cycles). It should be noted that all the comparisons between LGN and $\mathrm{LiNbO}_{3}$ are made at the same pump intensity of $10 \mathrm{GW} / \mathrm{cm}^{2}$ (for $0.1 \mathrm{~ns}$ pulses), which is close to the damage threshold of LGN. In practice, LiNbO3 cannot afford such high pump intensity, so its amplification performance might have been overestimated.

Moreover, another potential advantage of LGN is the extended IR transparent region. In $\mathrm{LiNbO}_{3}$ and $\mathrm{KTA}$, strong absorption will arise for the wavelength beyond $4 \mu \mathrm{m}$. In LGN, however, this wavelength limit is pushed to $\sim 6 \mu \mathrm{m}$. This undoubtedly enlarges the achievable operation range of intense mid-IR OPCPAs. In summary, LGN is a very qualified candidate for the nonlinear crystals of OPA and/or OPCPA aiming to obtain high-peak-power few-cycle mid-IR pulses.

4.3. $\mathrm{La}_{3} \mathrm{Ga}_{5.5} \mathrm{Ta}_{0.5} \mathrm{O}_{14}$ (LGT). In 2014, Boursier's group conducted a comprehensive study of the second-harmonic generation (SHG), frequency generation (SFG), and difference frequency generation (DFG) characteristics of the LGT over the entire transparency range [22]. The transmission range of LGN is $0.3 \sim 6.5 \mu \mathrm{m}$. The nonlinear coefficient $d_{11}$ of LGN was $2.4 \pm 0.4 \mathrm{pm} / \mathrm{V}$ at $670 \mathrm{~nm}$. Moreover, the optical damage threshold of LGN is $4.34 \mathrm{GW} / \mathrm{cm}^{2}$ at $1064 \mathrm{~nm}$, which is bigger than that of KTP, $\mathrm{LiNbO}_{3}$, and LGN.

They used the sphere method for the phase-matching studies. By shaping the LGT crystal as a sphere with a diameter of $4.70 \mathrm{~mm}$ (insert of Figure 11(a)), the SHG, SFG, and DFG phase-matching curves were measured as a function of the phase-matching angle $\theta_{\mathrm{PM}}$, as shown in Figure 11. Clearly, the interpolated tuning curves are much better in agreement with the experimental data than the calculations based on Sellmeier equations. 


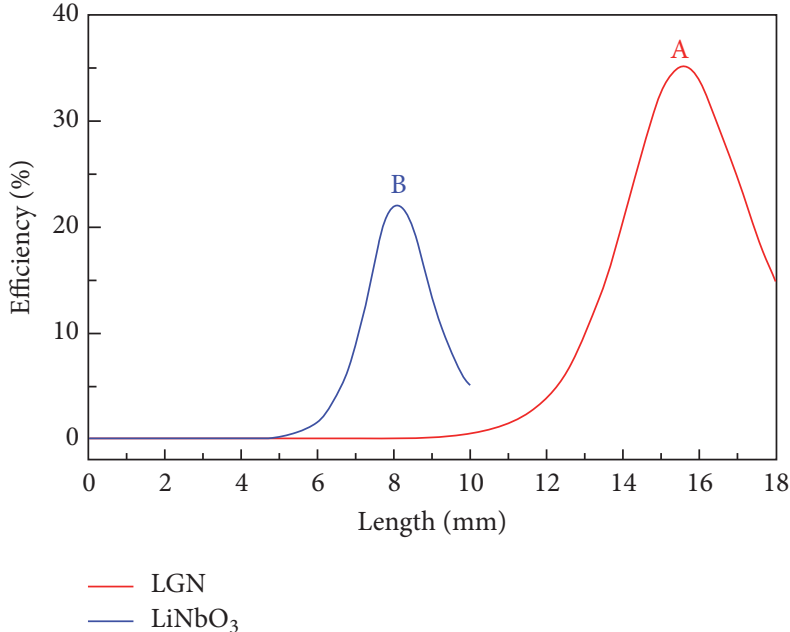

(a)

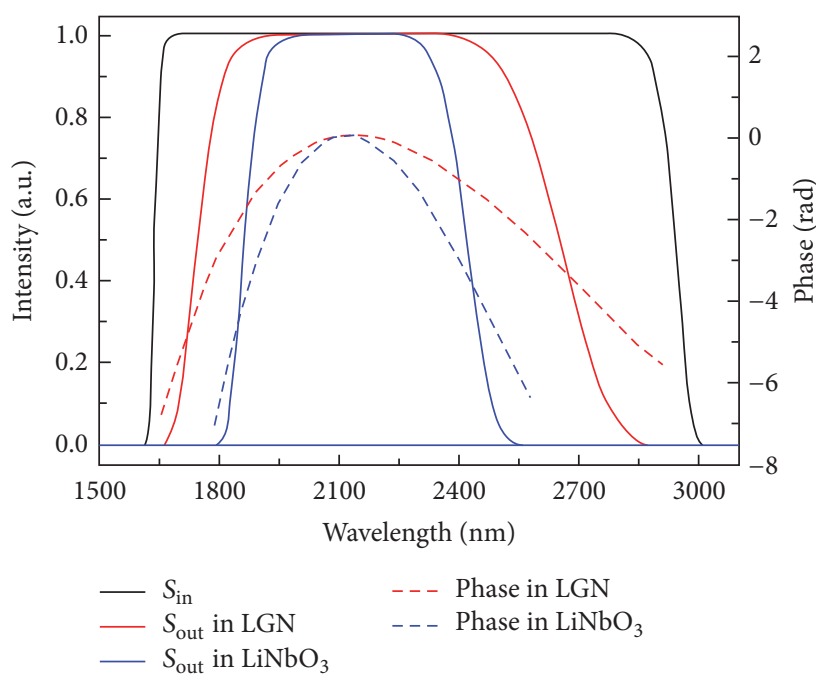

(c)

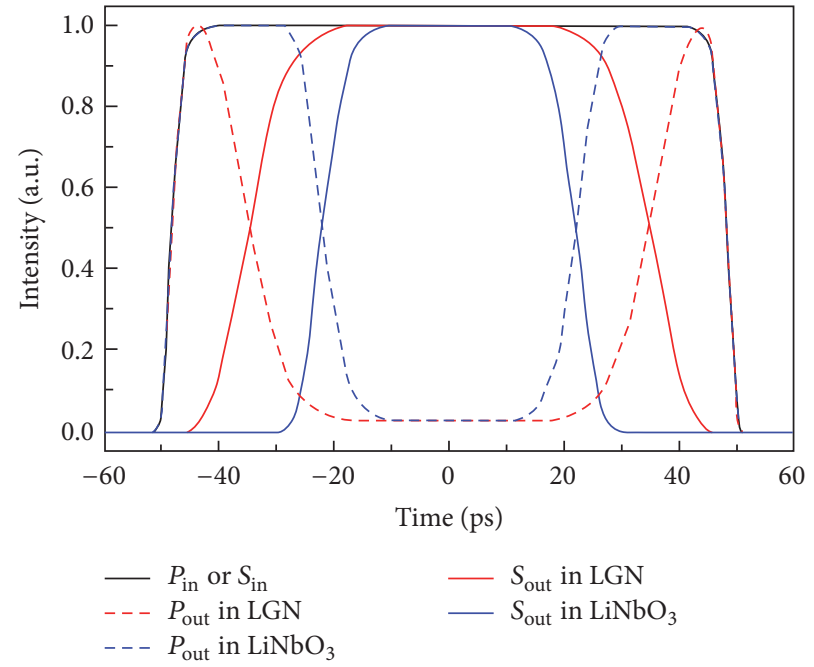

(b)

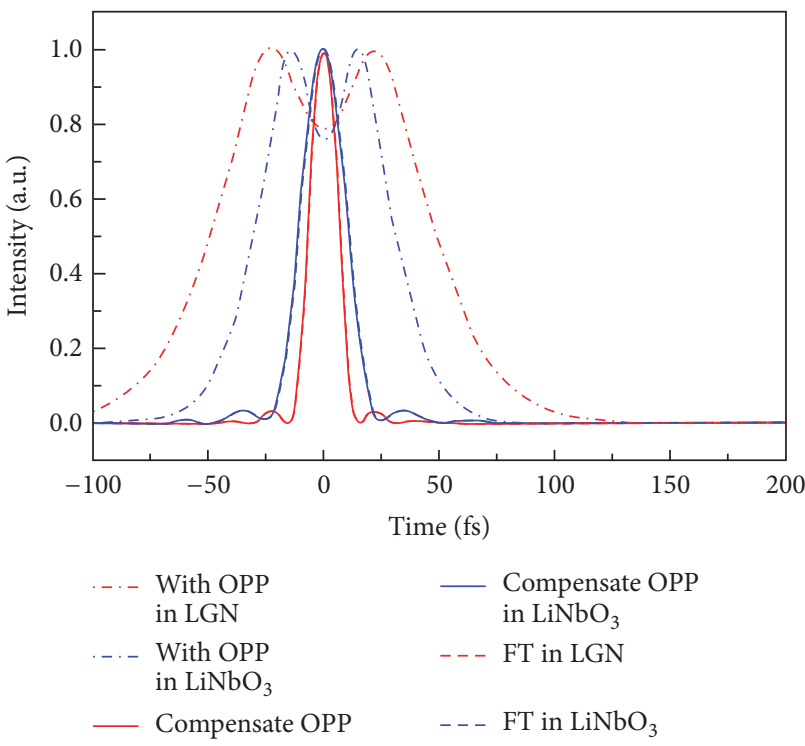

(d)

FIGURE 10: Simulation results for the Type I degenerate OPCPA pumped at $1.054 \mu \mathrm{m}$. (a) Efficiency evolution along the crystal length in LGN (red curve) and $\mathrm{LiNbO}_{3}$ (blue curve). (b) Temporal profiles of the input pump or chirped signal pulses, the residual pump pulses, and the amplified chirped signal pulses in $\mathrm{LGN}$ and $\mathrm{LiNbO}_{3}$. (c) Spectra of the input signal and the amplified signals in $\mathrm{LGN}_{\text {and }} \mathrm{LiNbO}$. The red and blue dashed curves represent the OPP produced in LGN- and $\mathrm{LiNbO}_{3}$-based OPCPAs, respectively. (d) The compressed pulses without and with OPP compensation in LGN- and $\mathrm{LiNbO}_{3}$-based OPCPAs. The dashed curves represent the corresponding Fourier-transform-limited pulses. Reprinted with permission from [21]. Copyright 2016 Optical Society of America.

The experimental results show that LGT is able to generate up to $6.5 \mu \mathrm{m}$ by tuning the phase-matching angle in the Type II configuration. All these good indicators put LGT as a new promising nonlinear crystal for the near-infrared range, especially above $2 \mu \mathrm{m}$ that covers Band II of transparency of the atmosphere. Furthermore, LGT can be grown to about $10 \mathrm{~cm}$ with very high purity, enabling us to work with laser beams of large diameter that permits working at high energies while remaining below the damage threshold power density of the crystal.

\section{Conclusions and Outlook}

In summary, we review the latest applications of langasite crystals in nonlinear optics. From the beginning of the application of LGS piezoelectrics, until now, the application of the crystal in electrooptical and nonlinear fields is developing rapidly. The structure and properties of LGS, LGN, and LGT are mainly of concern. Their broad transparent region, moderate effective nonlinear coefficient, high damage threshold, large size scalability, and good thermomechanical properties 

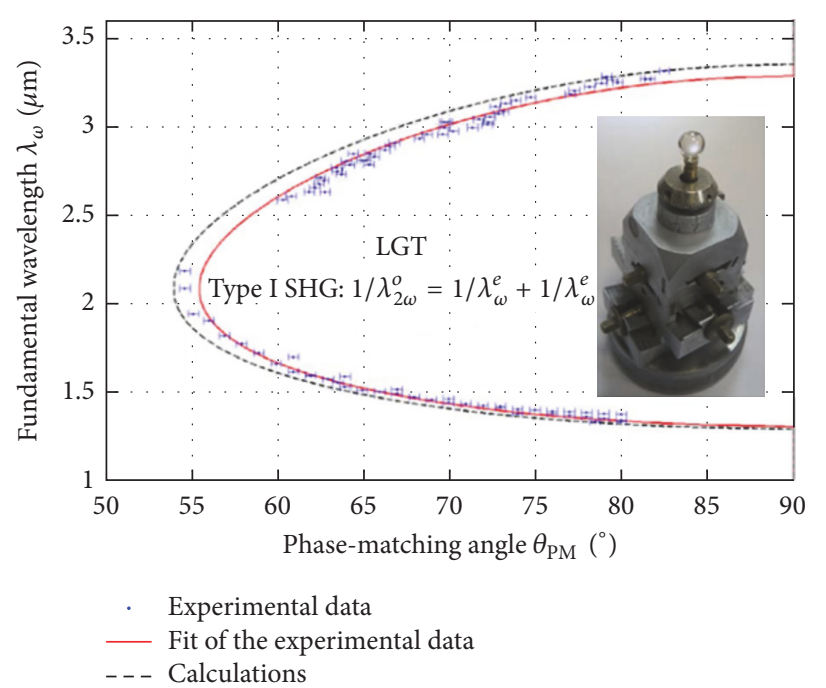

(a)

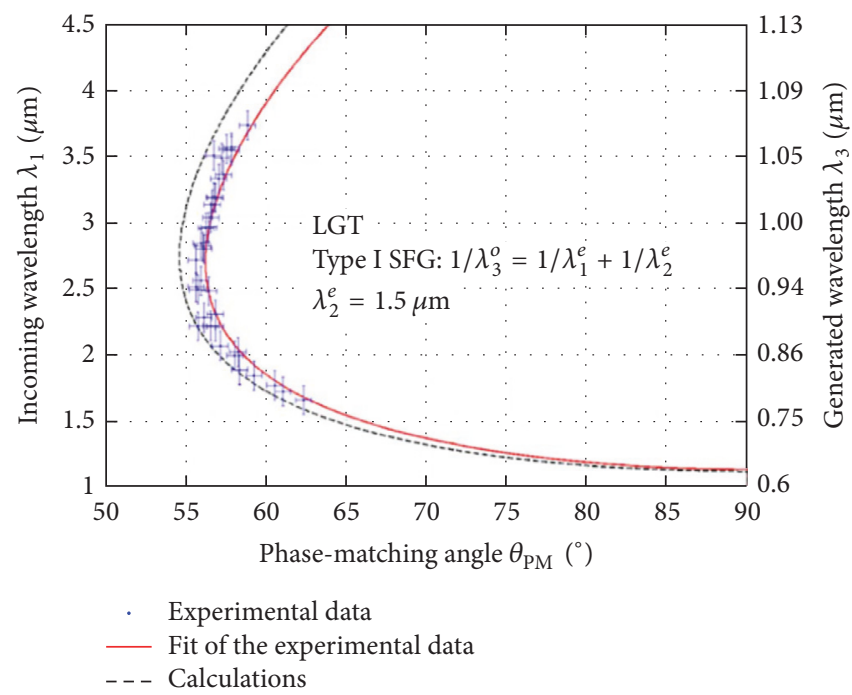

(b)

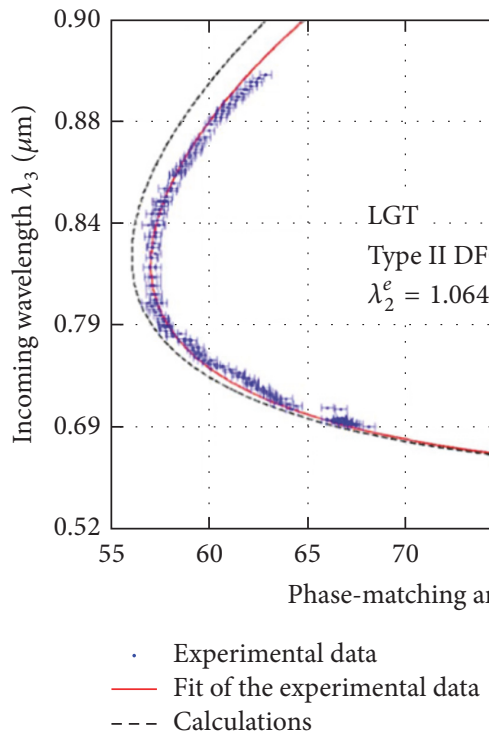

(c)

FIGURE 11: (a) Type I SHG tuning curve of LGT. $\lambda_{\omega}$ is plotted as a function of the phase-matching angle $\theta_{\mathrm{PM}}$. The insert picture shows the $4.70 \mathrm{~mm}$ diameter LGT sphere stuck on a goniometric head. (b) Type I SFG tuning curve of LGT. $\lambda_{1}$ and $\lambda_{3}$ are plotted as a function of the phase-matching angle $\theta_{\mathrm{PM}}$. (c) Type II DFG tuning curve of LGT. $\lambda_{3}$ and $\lambda_{1}$ are plotted as a function of the phase-matching angle $\theta_{\mathrm{PM}}$. Reprinted with permission from [22]. Copyright 2014 Optical Society of America.

make them very promising as a kind of mid-IR nonlinear crystal. The properties of the langasite family of compounds are summarized in Table 2.

Although much progress has been made in mid-IR NLO materials, there is still a lot of work to be done. Researchers should pay attention to the following aspects:

(i) Growing large-size crystals: although many new midIR materials possess excellent SHG properties, largesize crystals for practical applications have not been obtained [16]. Many important physical measurements require large-size crystals, including IR absorption edge, LDT value, and SHG tensor component. So, it is necessary to grow large-size crystals for eventually evaluating the practical application prospect of new NLO materials. According to a thorough survey in inorganic crystal structure database (ISCD), there are more than 30 compounds which belong to langasite family. However, the large-size candidates are not more than 10 . Therefore, researchers should pay more attention to crystal growth.

(ii) Discovering the relationship between the structure and properties of the material: up to now, it is still unclear how the microscopic structures accurately affect the IR absorption edges, birefringence dispersion, and LDT values. In particular, the latter two properties may play a crucial role in achieving 
TABLE 2: Comparison of the physical parameters of the LGS, LGN, and LGT.

\begin{tabular}{|c|c|c|c|}
\hline & LGS & LGN & LGT \\
\hline Point group & 32 & 32 & 32 \\
\hline \multirow{2}{*}{ Lattice parameters, $\AA$} & $a=8.170$ & $a=8.233$ & $a=8.236$ \\
\hline & $c=5.098$ & $c=5.129$ & $c=5.128$ \\
\hline \multicolumn{4}{|l|}{ Thermal conductivity, $\mathrm{W}(\mathrm{m} \cdot \mathrm{K})^{-1}$} \\
\hline$X$-direction & 1.3 & 1.4 & 1.2 \\
\hline$Z$-direction & 1.9 & 1.7 & 1.7 \\
\hline \multirow{2}{*}{ Thermal expansion coefficient $\left(10^{-6} / \mathrm{K}\right)$} & $\alpha_{11}=5.8$ & $\alpha_{11}=6.1$ & $\alpha_{11}=5.5$ \\
\hline & $\alpha_{33}=3.9$ & $\alpha_{33}=4.8$ & $\alpha_{33}=4.4$ \\
\hline Specific heat, $J /(g \cdot K)$ & 0.45 & 0.50 & 0.36 \\
\hline LDT (GW/cm²)@1.064 $\mu \mathrm{m}$ & 0.90 & 1.41 & 4.34 \\
\hline Transmission range $(\mu \mathrm{m})$ & $0.24 \sim 5.0$ & $0.28 \sim 7.4$ & $0.3 \sim 6.8$ \\
\hline$n_{o}(@ 1.083 \mu \mathrm{m})$ & 1.88021 & 1.92706 & 1.91745 \\
\hline$n_{e}(@ 1.083 \mu \mathrm{m})$ & 1.89156 & 1.95623 & 1.94338 \\
\hline Birefringence @1.083 $\mu \mathrm{m}$ & 0.01135 & 0.02917 & 0.02593 \\
\hline \multirow[t]{2}{*}{ EO coefficient $(\mathrm{pm} / \mathrm{V})$} & $\gamma_{11}=-2.68$ & $\gamma_{11}=-2.62$ & $\gamma_{11}=-2.82$ \\
\hline & $\gamma_{41}=1.22$ & $\gamma_{41}=0.85$ & $\gamma_{41}=0.75$ \\
\hline SHG coefficient (pm/V) @ $0.532 \mu \mathrm{m}$ & $d_{11}=1.7$ & $d_{11}=2.6$ & $d_{11}=2.3$ \\
\hline
\end{tabular}

high-power harmonic output. For langasite family, there are many investigations about the relationship between piezoelectricity and chemical composition. However, the NLO properties have long been overlooked. The respective contribution to linear and nonlinear optical properties of each cation-centered polyhedron should be elucidated. And then, some excellent materials would be designed on the basis of this clear map. We believe that high-throughput DFT calculations would be very suitable for this case.

(iii) Exploring the new types of metal oxides that can be transparent to mid-IR region: generally, heavy atoms should be given precedence owing to their low frequency phonon vibrations. On the other hand, the bandgap of the designed materials should maintain a large value, which is favorable to obtain high LDT. We believe that these investigations would pave the way for new mid-IR NLO materials.

\section{Conflicts of Interest}

The authors declare no conflicts of interest.

\section{Acknowledgments}

This work was supported by the National Natural Science Foundation of China (nos. 51025210, 51102156, 51422205, and 51272131), the Natural Science Foundation for Distinguished Young Scholars of Shandong Province (2014JQE27019), and Taishan Scholar Foundation of Shandong Province, China.

\section{References}

[1] J. D. Bierlein and H. Vanherzeele, "Potassium titanyl phosphate: properties and new applications," Journal of the Optical Society of America B: Optical Physics, vol. 6, no. 4, pp. 622-633, 1989.
[2] C. T. Chen, B. C. Wu, A. D. Jiang, and G. M. You, "A new-type ultraviolet SHG crystal- $\beta$-BaB2O4," Science in China Series BChemistry, vol. 28, no. 3, pp. 235-243, 1985.

[3] C. Chen, Y. Wu, and A. Jiang, "New nonlinear-optical crystal: $\mathrm{LiB}_{3} \mathrm{O}_{5}$," Journal of the Optical Society of America B: Optical Physics, vol. 6, no. 4, pp. 616-621, 1989.

[4] C. Chen, J. Lu, T. Togashi et al., "Second-harmonic generation from a $\mathrm{KBe}_{2} \mathrm{BO}_{3} \mathrm{~F}_{2}$ crystal in the deep ultraviolet," Optics Expresss, vol. 27, no. 8, pp. 637-639, 2002.

[5] C. T. Chen, G. L. Wang, X. Y. Wang, and Z. Y. Xu, "Deep$\mathrm{UV}$ nonlinear optical crystal $\mathrm{KBe}_{2} \mathrm{BO}_{3} \mathrm{~F}_{2}$-discovery, growth, optical properties and applications," Applied Physics B: Lasers and Optics, vol. 97, no. 1, pp. 9-25, 2009.

[6] M. Ghotbi, Z. Sun, A. Majchrowski, E. Michalski, I. V. Kityk, and M. Ebrahim-Zadeh, "Efficient third harmonic generation of microjoule picosecond pulses at $355 \mathrm{~nm}$ in $\mathrm{BiB}_{3} \mathrm{O}_{6}$," Applied Physics Letters, vol. 89, no. 17, Article ID 173124, 2006.

[7] V. Petrov, "Frequency down-conversion of solid-state laser sources to the mid-infrared spectral range using non-oxide nonlinear crystals," Progress in Quantum Electronics, vol. 42, pp. 1-106, 2015.

[8] A. Arriola, S. Gross, M. Ams et al., "Mid-infrared astrophotonics: study of ultrafast laser induced index change in compatible materials," Optical Materials Express, vol. 7, no. 3, pp. 698-711, 2017.

[9] Z. Zhao, B. Wu, X. Wang et al., "Mid-infrared supercontinuum covering $2.0-16 \mu \mathrm{m}$ in a low-loss telluride single-mode fiber," Laser \& Photonics Reviews, vol. 11, no. 2, Article ID 1700005, 2017.

[10] D. Pestov, X. Wang, G. O. Ariunbold et al., "Single-shot detection of bacterial endospores via coherent Raman spectroscopy," Proceedings of the National Acadamy of Sciences of the United States of America, vol. 105, no. 2, pp. 422-427, 2008.

[11] I. Chung and M. G. Kanatzidis, "Metal chalcogenides: A rich source of nonlinear optical materials," Chemistry of Materials, vol. 26, no. 1, pp. 849-869, 2014. 
[12] M. C. Ohmer, R. Pandey, and B. H. Bairamov, "Emergence of chalcopyrites as nonlinear optical materials," MRS Bulletin, vol. 23, no. 7, pp. 16-20, 1998.

[13] W. Ruderman, J. Maffetone, D. E. Zelman, and D. M. Poirier, "Laser damage studies of silver gallium sulfide single crystals," MRS Online Proceedings Library, vol. 484, article 519, 1997.

[14] P. G. Schunemann, "Crystal growth and properties of nonlinear optical materials," in Perspectives on Inorganic, Organic, and Biological Crystal Growth: From Fundamentals to Applications, vol. 916, pp. 541-559, American Institute of Physics, College Park, Md, USA, 2007.

[15] F. Liang, L. Kang, Z. Lin, Y. Wu, and C. Chen, "Analysis and prediction of mid-IR nonlinear optical metal sulfides with diamond-like structures," Coordination Chemistry Reviews, vol. 333, pp. 57-70, 2017.

[16] F. Liang, L. Kang, Z. Lin, and Y. Wu, "Mid-infrared nonlinear optical materials based on metal chalcogenides: structureproperty relationship," Crystal Growth \& Design, vol. 17, no. 4, pp. 2254-2289, 2017.

[17] D. Lu, T. Xu, H. Yu et al., "Acentric langanite $\mathrm{La}_{3} \mathrm{Ga}_{5.5} \mathrm{Nb}_{0.5} \mathrm{O}_{14}$ crystal: a new nonlinear crystal for the generation of midinfrared parametric light," Optics Express, vol. 24, no. 16, pp. 17603-17615, 2016.

[18] S. Ma, H. Yu, H. Zhang et al., "Efficient high repetition rate electro-optic Q-switched laser with an optically active langasite crystal," Scientific Reports, vol. 6, Article ID 30517, 2016.

[19] L. Wang, X. Cai, J. Yang, X. Wu, H. Jiang, and J. Wang, “520 mJ langasite electro-optically Q-switched Cr,Tm,Ho:YAG laser," Optics Expresss, vol. 37, no. 11, pp. 1986-1988, 2012.

[20] L. Wang, J. Wang, J. Yang et al., " $2.79 \mu \mathrm{m}$ high peak power LGS electro-optically Q-switched Cr,Er:YSGG laser," Optics Expresss, vol. 38, no. 12, pp. 2150-2152, 2013.

[21] J. Ma, J. Wang, D. Hu et al., "Theoretical investigations of broadband mid-infrared optical parametric amplification based on a $\mathrm{La}_{3} \mathrm{Ga}_{5.5} \mathrm{Nb}_{0.5} \mathrm{O}_{14}$ crystal," Optics Express, vol. 24, no. 21, pp. 23957-23968, 2016.

[22] E. Boursier, P. Segonds, B. Boulanger et al., "Phase-matching directions, refined Sellmeier equations, and second-order nonlinear coefficient of the infrared Langatate crystal $\mathrm{La}_{3} \mathrm{Ga}_{5.5} \mathrm{Ta}_{0.5} \mathrm{O}_{14}$, Optics Expresss, vol. 39, no. 13, pp. 40334036, 2014.

[23] H. Takeda, R. Aoyagi, S. Okamura, and T. Shiosaki, "Cation distribution and melting behavior of $\mathrm{La}_{3} \mathrm{Ga}_{5} \mathrm{M}^{4+} \mathrm{O}_{14}(\mathrm{M}=\mathrm{Si}$, Ti, Ge, Zr, Sn, and Hf) crystals," Ferroelectrics, vol. 295, no. 1, pp. 67-76, 2003.

[24] J. Stade, L. Bohatý, M. Hengst, and R. B. Heimann, “Electrooptic, piezoelectric and dielectric properties of langasite $\left(\mathrm{La}_{3} \mathrm{Ga}_{5} \mathrm{SiO}_{14}\right)$, langanite $\left(\mathrm{La}_{3} \mathrm{Ga}_{5.5} \mathrm{Nb}_{0.5} \mathrm{O}_{14}\right)$ and langataite $\left(\mathrm{La}_{3} \mathrm{Ga}_{5.5} \mathrm{Ta}_{0.5} \mathrm{O}_{14}\right)$," Crystal Research and Technology, vol. 37, no. 10, pp. 1113-1120, 2002.

[25] S. Zhang and F. Yu, "Piezoelectric materials for high temperature sensors," Journal of the American Ceramic Society, vol. 94, no. 10, pp. 3153-3170, 2011.

[26] D. Roshchupkin, L. Ortega, O. Plotitcyna et al., "Piezoelectric $\mathrm{Ca}_{3} \mathrm{NbGa}_{3} \mathrm{Si}_{2} \mathrm{O}_{14}$ crystal: crystal growth, piezoelectric and acoustic properties," Applied Physics A, vol. 122, no. 8, article 753, 2016.

[27] A. A. Kaminskii, I. M. Silvestrova, S. E. Sarkisov, and G. A. Denisenko, "Investigation of trigonal $\left(\mathrm{La}_{1-x} \mathrm{Nd}_{x}\right)_{3} \mathrm{Ga}_{5} \mathrm{SiO}_{14}$ crystals. II. Spectral laser and electromechanical properties," Physica Status Solidi (A)_Applications and Materials Science, vol. 80 , no. 2, pp. 607-620, 1983.
[28] A. A. Kaminskii, B. V. Mill, G. G. Khodzhabagyan, A. F. Konstantinova, A. I. Okorochkov, and I. M. Silvestrova, "Investigation of trigonal $\left(\mathrm{La}_{1-x} \mathrm{Nd}_{x}\right)_{3} \mathrm{Ga}_{5} \mathrm{SiO}_{14}$ crystals. I. Growth and optical Properties," Physica Status Solidi (A)-Applications and Materials Science, vol. 80, no. 1, pp. 387-398, 1983.

[29] A. A. Kaminskii, E. L. Belokoneva, B. V. Mill et al., "Pure and $\mathrm{Nd}^{3+}$-doped $\mathrm{Ca}_{3} \mathrm{Ga}_{2} \mathrm{Ge}_{4} \mathrm{O}_{14}$ and $\mathrm{Sr}_{3} \mathrm{Ga}_{2} \mathrm{Ge}_{4} \mathrm{O}_{14}$ single crystals, their structure, optical, spectral luminescence, electromechanical properties, and stimulated emission," Physica Status Solidi (A)_Applications and Materials Science, vol. 86, no. 1, pp. 345362, 1984.

[30] A. Pavlovska, J. Schneider, S. Werner, B. Maximov, B. Mill, and C. Baetz, "Thermal expansion of lattice parameters of $\mathrm{La}_{3} \mathrm{Nb}_{0.5} \mathrm{Ga}_{5.5} \mathrm{O}_{14}, \mathrm{La}_{3} \mathrm{Ta}_{0.5} \mathrm{Ga}_{5.5} \mathrm{O}_{14}$ and $\mathrm{La}_{3} \mathrm{SbZn}_{3} \mathrm{Ge}_{2} \mathrm{O}_{14}$," Zeitschrift für Kristallographie, vol. 218, no. 3, pp. 187-192, 2003.

[31] J. Wang, X. Yin, R. Han et al., "Growth, properties and electrooptical applications of single crystal $\mathrm{La}_{3} \mathrm{Ga}_{5} \mathrm{SiO}_{14}$," Optical Materials, vol. 23, no. 1-2, pp. 393-397, 2003.

[32] O. M. Kugaenko, S. S. Uvarova, S. A. Krylov et al., "Basic thermophysical parameters of langasite $\left(\mathrm{La}_{3} \mathrm{Ga}_{5} \mathrm{SiO}_{14}\right)$, langatate $\left(\mathrm{La}_{3} \mathrm{Ta}_{0.5} \mathrm{Ga}_{5.5} \mathrm{O}_{14}\right)$, and catangasite $\left(\mathrm{Ca}_{3} \mathrm{TaGa}_{3} \mathrm{Si}_{2} \mathrm{O}_{14}\right)$ single crystals in a temperature range of 25 to $1000^{\circ} \mathrm{C}$," Bulletin of the Russian Academy of Sciences, Physics, vol. 76, no. 11, pp. 12581263, 2012.

[33] A. A. Kaminskii, A. V. Butashin, I. A. Maslyanitsin, and V. D. Shigorin, "Nonlinear optical properties of acentric crystals with Ca-gallogermanate structure," Physica Status Solidi (A)_Applications and Materials Science, vol. 112, no. 1, pp. K49K52, 1989.

[34] L. Kang, S. Luo, H. Huang et al., "Prospects for fluoride carbonate nonlinear optical crystals in the UV and deep-UV regions," The Journal of Physical Chemistry C, vol. 117, no. 48, pp. 25684-25692, 2013

[35] A. F. Nieuwenhuis, C. J. Lee, P. J. M. Van Der Slot, I. D. Lindsay, P. Groß, and K.-J. Boller, "High-efficiency mid-infrared $\mathrm{ZnGeP2}$ optical parametric oscillator directly pumped by a lamppumped, Q-switched CrTmHo:YAG laser," Optics Expresss, vol. 33, no. 1, pp. 52-54, 2008.

[36] H. Kong, J. Wang, H. Zhang et al., "Growth, properties and application as an electrooptic Q-switch of langasite crystal," Journal of Crystal Growth, vol. 254, no. 3-4, pp. 360-367, 2003.

[37] H. Tang, X. Zhu, and Y. Feng, "Comparison of $30 \mathrm{kHz}$ Qswitched Nd:YVO4 lasers with LGS and RTP electro-optic modulator," in Proceedings of the 8th Pacific Rim Conference on Lasers and Electro-Optics (CLEO/Pacific Rim '09), Shanghai, China, August 2009.

[38] L. A. W. Gloster, P. Cormont, A. M. Cox, T. A. King, and B. H. T. Chai, "Diode-pumped Q-switched Yb:S-FAP laser," Optics Communications, vol. 146, no. 1-6, pp. 177-180, 1998.

[39] Y. H. Chen and Y. C. Huang, "Actively Q-switched Nd:YVO4 laser using an electro-optic periodically poled lithium niobate crystal as a laser Q-switch," Optics Expresss, vol. 28, no. 16, pp. 1460-1462, 2003.

[40] Q. Liu, M. Gong, H. Wu, F. Lu, and C. Li, "Electro-optic Qswitched Yb:YAG slab laser," Laser Physics Letters, vol. 3, no. 5, pp. 249-251, 2006.

[41] G. Hansson, H. Karlsson, S. Wang, and F. Laurell, "Transmission measurements in KTP and isomorphic compounds," Applied Optics, vol. 39, no. 27, pp. 5058-5069, 2000.

[42] R. Komatsu, T. Sugawara, and S. Uda, "Nonlinear optical properties of langasite crystal," Japanese Journal of Applied Physics, vol. 36, no. 9, pp. 6159-6161, 1997. 
[43] N. Thiré, S. Beaulieu, V. Cardin et al., " $10 \mathrm{~mJ}$ 5-cycle pulses at $1.8 \mu \mathrm{m}$ through optical parametric amplification," Applied Physics Letters, vol. 106, no. 9, Article ID 091110, 2015.

[44] A. V. Mitrofanov, A. A. Voronin, S. I. Mitryukovskiy et al., "Mid-infrared-to-mid-ultraviolet supercontinuum enhanced by third-to-fifteenth odd harmonics," Optics Expresss, vol. 40, no. 9, pp. 2068-2071, 2015. 

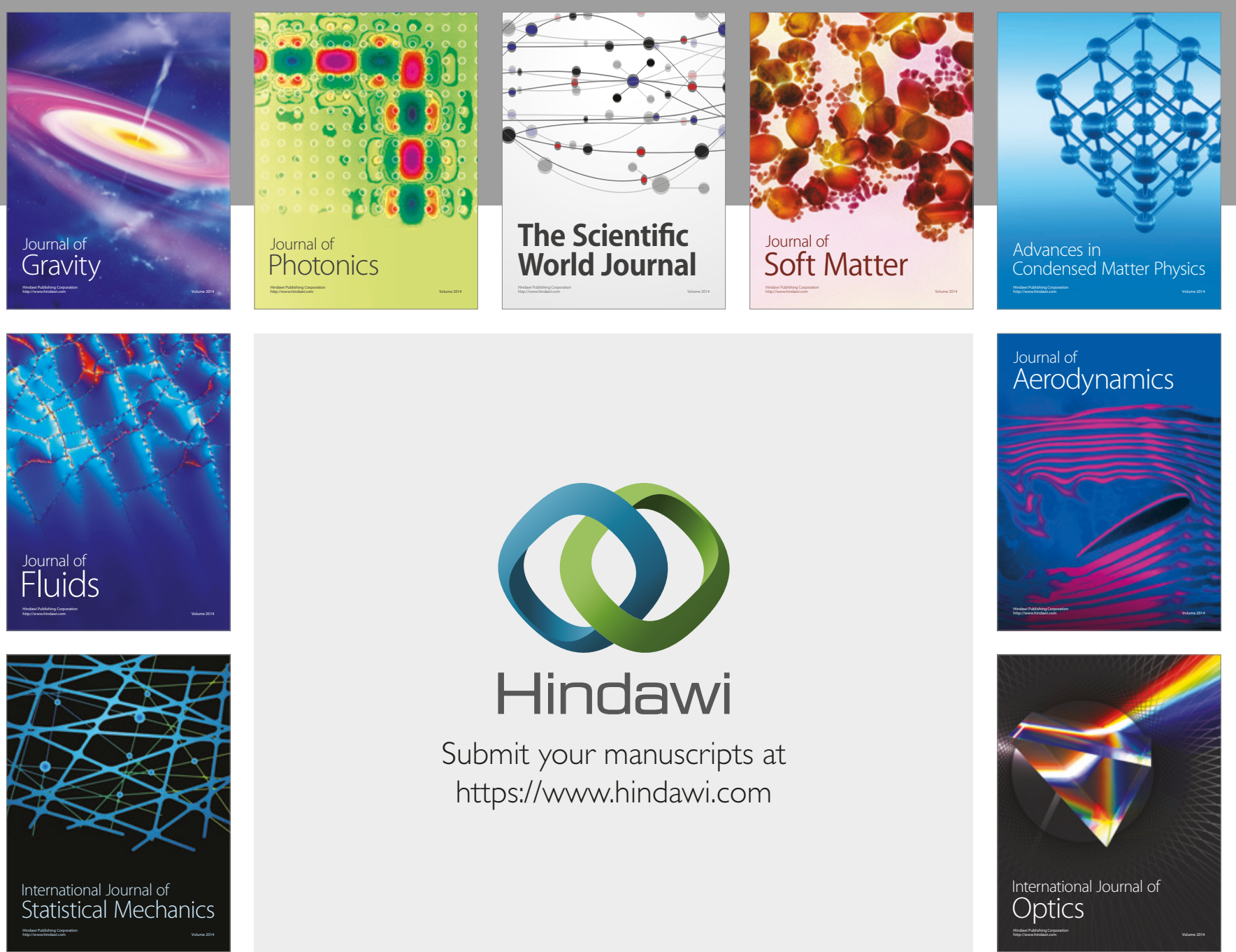

Submit your manuscripts at

https://www.hindawi.com
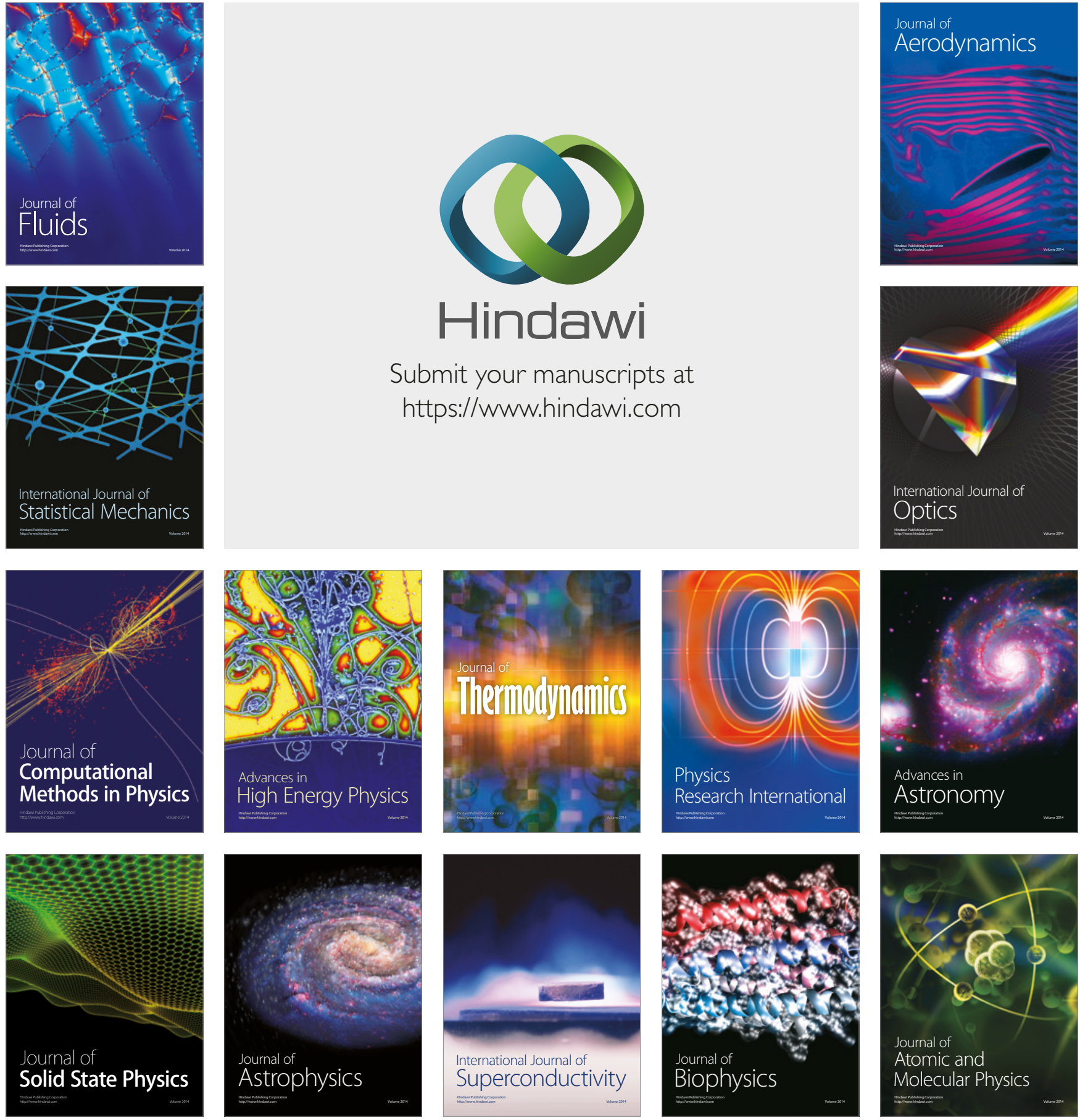